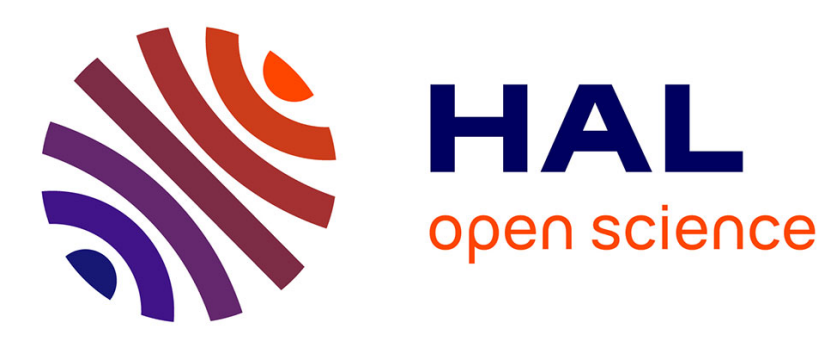

\title{
Predictive maintenance policy for a gradually deteriorating system subject to stress
}

Estelle Deloux, Bruno Castanier, Christophe Bérenguer

\section{To cite this version:}

Estelle Deloux, Bruno Castanier, Christophe Bérenguer. Predictive maintenance policy for a gradually deteriorating system subject to stress. Reliability Engineering and System Safety, 2009, pp.418-431. 10.1016/j.ress.2008.04.002 . hal-00361288

\section{HAL Id: hal-00361288 \\ https://hal.science/hal-00361288}

Submitted on 17 Feb 2009

HAL is a multi-disciplinary open access archive for the deposit and dissemination of scientific research documents, whether they are published or not. The documents may come from teaching and research institutions in France or abroad, or from public or private research centers.
L'archive ouverte pluridisciplinaire HAL, est destinée au dépôt et à la diffusion de documents scientifiques de niveau recherche, publiés ou non, émanant des établissements d'enseignement et de recherche français ou étrangers, des laboratoires publics ou privés. 


\title{
Predictive maintenance policy for a gradually deteriorating system subject to stress
}

\author{
E. Deloux ${ }^{a}$, B. Castanier ${ }^{a}$, C.Bérenguer ${ }^{b}$ \\ ${ }^{\mathrm{a}}$ IRCCyN/Ecole des Mines de Nantes, Nantes, France \\ ${ }^{\mathrm{b}}$ Université de Technologie de Troyes/CNRS, Troyes, France
}

\begin{abstract}
This paper deals with a predictive maintenance policy for a continuously deteriorating system subject to stress. We consider a system with two failure mechanisms which are respectively due to an excessive deterioration level and a shock. To optimize the maintenance policy of the system, an approach combining Statistical Process Control (SPC) and Condition-Based Maintenance (CBM) is proposed. CBM policy is used to inspect and replace the system according to the observed deterioration level. SPC is used to monitor the stress covariate. In order to assess the performance of the proposed maintenance policy and to minimize the long-run expected maintenance cost per unit of time, a mathematical model for the maintained system cost is derived. Analysis based on numerical results are conducted to highlight the properties of the proposed maintenance policy in respect to the different maintenance parameters.
\end{abstract}

Key words: predictive maintenance, stochastic modeling, control chart, economic performance

\section{Introduction}

Many manufacturing processes or structural systems suffer increasing wear with usage or age and are subject to random failures resulting from this deterioration [1] and most of them are maintained or repairable systems. Moreover, for some systems, such as aircrafts, submarines, military systems, and nuclear systems, it is very important to avoid failure during actual operation because it can be dangerous or disastrous. Therefore, maintenance of them is necessary since it can improve reliability. The growing importance of maintenance has generated an increasing interest in the development and implementation of optimal maintenance strategies for improving system availability, preventing 
the occurrence of system failures, and reducing maintenance costs of deteriorating systems. A lot of models for optimizing the maintenance scheduling and planning have been proposed [2]. Maintenance can be categorized into two major classes: corrective and preventive. Preventive maintenance itself can be categorized into two classes: systematic preventive maintenance and Condition-Based Maintenance (CBM). CBM approaches are usually more efficient than systematic preventive maintenance policies based on the a priori statistical knowledge of the system lifetime [3]. CBM policies are thus particularly justified for critical systems and the collected information on the system condition can be exploited by the maintenance decision-maker in order to maximize the availability of the system and to minimize its long-run expected cost. The price for this higher efficiency is an enhanced modeling work of the maintained system behavior in order to support correctly the maintenance decisions [4].

A classical assumption in CBM modeling is that the system failure can be explained by a deterioration process. One way to model continuous and gradual deterioration process due to wear and tear, e.g. systems subject to erosion (hydraulic structures, dikes) [5], consumption [6], cumulative wear (cutting tools) [7], is to represent the time-dependent deterioration by single continuous state stochastic process. The failure occurs when this deterioration exceeds a failure threshold [8]. But, in fact, because of the complexity of the systems and the influence of different variables and stresses $[9,10]$ on the failure mechanism, it is difficult, in most of the cases, to reduce failure mechanisms to one deterioration process. Nevertheless, partial information on the system state can be tackled by the monitoring of easily-observable covariates (e.g. vibration, temperature, humidity,...). Some works focused on non-maintained system reliability, propose relevant degradation models [11] such as hazard rate processes [12-15], competing risk processes [16] for example. However, only few are developed in such a context for CBM applications [17].

Moreover, a system failure can also induce some changes in the environmental conditions and especially in the previously mentioned easily observable covariates. For example, the internal vibration signal can be influenced by the present of springlers in a ball-bearing. Hence, the observation of such covariates should provide some partial information of the system state. Condition Monitoring (CM) received a great deal of attention in literature $[18,19]$. CM methods, e.g. Statistical Process Control (SPC), applied to systems are used to detect potential failures or to determine updated failure rate values of sensitive components. For example, vibration analysis, oil analysis, humidity, temperature and so forth are variables allowing the detection of a failure, because the stress intensity enhances when a failure has occurred, but also the acceleration of the failure mechanisms due to stress. The use of CM techniques will generally improve plant production availability, and reduces downtime costs. Finally, modern maintenance paradigm consists of two major elements: preventive maintenance and condition monitoring decision analysis. Both of these have received significant development in recent decades but a longstand- 
ing gap continues to exist between these two elements. However, coordinate SPC and preventive maintenance presents economic performance [20-23]. Cassady et al. [21] performed a preliminary investigation to model and analyze the relationship between maintenance and quality combining classical agebased approaches and SPC. Ben-Daya and Rahim [20] provide a framework for capturing maintenance and quality control interactions leading to models for their joint optimization. Linderman et al. [23] develop an analytical model to demonstrate this economic benefit.

In this study, we propose to answer to the different points presented previously, in particular the proposition of a model for a deteriorating system subject to stress and the construction and evaluation of a maintenance policy for such a model. More precisely, we consider a system with two failure mechanisms which are due to an excessive deterioration level and a shock due to a stressful environment. We propose here to model the failure process by the introduction of two explicative variables, a cumulative deterioration process, which is modeled by a non-decreasing stochastic process, and a stress covariate, which modeled by a stochastic process fluctuating around a given mean. To optimize the maintenance policy of the system, a combined SPC and CBM approach is proposed. CBM is used to inspect and replace the system according to the observed deterioration level. SPC is used to monitor the stress covariate. In this study, we develop a model which allows us to investigate the influence of the maintenance decision parameters on the total cost of the maintained system and we show that the long-run expected maintenance cost per unit of time can be minimize by an appropriate joint choice of these decision variables. Furthermore, we perform a sensitivity analysis to highlight the properties of the optimal maintenance policies when maintenance data and system deterioration characteristics change.

This paper is organized as follows. In section 2, the failure process and the associated variables are described. Section 3 is devoted to the presentation of the maintenance policy. In section 4 , results from numerical experiments illustrate and analyze the behavior of the proposed maintenance policy. Finally, in Section 4, conclusions are drawn from the work. 


\begin{tabular}{|c|c|}
\hline \\
\hline \multicolumn{2}{|c|}{$\begin{array}{l}\text { Nomenclature } \\
\left(X_{k}\right)_{k \in \mathbb{N}}\end{array}$} \\
\hline$\left(Y_{k}\right)_{k \in \mathbb{N}}$ & discrete time process describing a stress at time $t_{k}$ \\
\hline$\left(Z_{k}\right)_{k \in \mathbb{N}}$ & discrete time process describing the system state at time $t_{k}$ \\
\hline$\Delta t$ & unit time length \\
\hline$t_{k}$ & discrete time \\
\hline$a$ & scale parameter \\
\hline$F_{A}$ & failure acceleration factor due to stress \\
\hline$b$ & sensitivity to stress \\
\hline$L$ & cumulative degradation threshold \\
\hline$m$ & nominal level of $Y_{t}$ \\
\hline$\alpha$ & deterioration parameter \\
\hline$\sigma$ & standard deviation of $Y_{t}$ \\
\hline$\mu$ & drift coefficient \\
\hline$\lambda$ & stress threshold \\
\hline$\xi$ & preventive replacement threshold \\
\hline$U C L$ & upper control limit \\
\hline$\tau$ & $\mathrm{X}$-inspection period \\
\hline$\delta$ & routine maintenance action period \\
\hline$\theta$ & maintenance data vector \\
\hline$\Xi$ & maintenance decision parameter vector \\
\hline$c_{i x}$ & cost per inspection of $X_{t}$ \\
\hline$c_{i z}$ & minimal inspection cost \\
\hline$c_{c}$ & cost per corrective replacement \\
\hline$c_{p}$ & cost per preventive replacement \\
\hline$c_{m}$ & greasing cost \\
\hline$c_{u}$ & unavailability cost \\
\hline$N_{i x}\left(t_{k}\right)$ & number of planned inspections \\
\hline$N_{i z}\left(t_{k}\right)$ & number of unplanned inspections \\
\hline$N_{m}\left(t_{k}\right)$ & number of planned routine maintenance \\
\hline$N_{p}\left(t_{k}\right)$ & number of preventives replacements \\
\hline$N_{c}\left(t_{k}\right)$ & number of correctives replacements \\
\hline$D_{u}\left(t_{k}\right)$ & cumulative unavailability duration \\
\hline$f_{\lambda}^{(k)}$ & probability density function of the deterioration \\
\hline & increment after $k$ periods of time of a non-failed system \\
\hline$r_{k}$ & time since the last routine maintenance \\
\hline
\end{tabular}




\section{Description of the combined stress-degradation failure process}

This section is devoted to describe the system failure process, the relationship between the stress and the system degradation behavior. Then, the associated mathematical model is derived.

\subsection{Description of the system failure process}

We consider a single-unit system subject to two failure mechanisms. The first failure process results from an excessive deterioration level. The second one is a lethal shock failure process which can be explained, at the same time, by the deterioration level and the stressful environment [14].

In order to illustrate our model, we will rest our various assumptions on the analysis of the particular failure mechanisms of a guiding system of a tramway due to chippings and vibrations.

\subsubsection{Cumulative deterioration failure}

The first failure mechanism results from an excessive deterioration level which can be described by a measurable variable. The system stops to fulfill its function as soon as the aging variable is greater than a predetermined level $L$. In this case, either a "hard" failure or a "soft" failure has occurred, viz. an important deterioration is present which reduces too much the system performance and the system is considered as failed, even if it is still working from e.g. a mechanical point of view. For example, a production system will continue to produce even if its performance level is not good enough. However, the resulting products will be of poor quality and should be scrapped and in this case the system can be considered in the failed state. The threshold $L$, in this example, represents the minimum level of system performance in order to obtain products conform to requirements. The determination of this threshold rests on a level of expertise and data related to nonconformities. Within the framework of the safety, $L$ is a tolerance which can be imposed directly by a regulation, for example, a wear threshold for tyres and brake pads. Furthermore a failure is assumed to be not self-announcing, for example, a bridge, a dike, ... may not be able to sustain its nominal load any longer, but an inspection is necessary to reveal it. Obviously, it is not reasonable to wait for the structure to collapse before declaring that it failed; it is in a failed state as soon as it can no longer fulfill its intended function under a given performance level. The threshold $L$ can thus be seen as a deterioration level which must not be exceeded for economical or security reasons. An inspection is needed in order to detect if the deterioration level is greater than this threshold $L$. 
Let $\left(X_{k}\right)_{k \in \mathbb{N}}$ be the continuous increasing stochastic process describing the deterioration process on an infinite discrete time $\left(t_{k}=k \Delta t\right)_{k \in \mathbb{N}}$. Later in this paper, the value of the unit time length $\Delta t$ will be fixed to 1 . We assume that $\left(X_{k}\right)_{k \in \mathbb{N}}$ is modeled by a phase-type distribution with parameter $\alpha$, i.e. an increment over one fixed time unit, $X_{k-1}-X_{k}$, is exponentially distributed with parameter $\alpha$ and a probability density function $f(x)=\alpha e^{-\alpha x}$. This model is classical and generally well-adapted to cumulative wear, fatigue, crack growth, corrosion,..., with a minimal information on the considered deterioration process [24].

The process $\left(X_{k}\right)_{k \in \mathbb{N}}$ represents in the example of the rollers, the propagation of the chipping.

\subsection{2 "Stress-failure" process}

The second failure mechanism is related to a stress-failure process. Let assume the system is subject to an environmental stress that can be external to the system (temperature, humidity, etc.) or a direct consequence of the system operating mode (internal vibrations, internal temperature, etc.). Let $\left(Y_{k}\right)_{k \in \mathbb{N}}$ be the associated stress process where $Y_{k}$ represents the stress intensity at time $t_{k} .\left(Y_{k}\right)$ is assumed to be a classical Gaussian process with a given mean $m$ and standard deviation $\sigma$.

The stress-failure process is modelled by a classical shock process, a failure can occur because of the stress with a given probability $1-q$. The associated stress-deterioration failure probability $1-q$ depends on the stress intensity $Y_{k}$. To model the influence of the stress on this probability, a stress threshold $\lambda$ is introduced. If $Y_{k}<\lambda$, the system is considered in the nominal operating state and the stress has a low impact on the probability of a failure. On the other hand, when $Y_{k} \geq \lambda$, the system is considered stressed and this increases the probability of a failure due to the stress. Let $F_{A}$ be the associated failure acceleration factor due to stress. Moreover, because the sensitivity of the system to stress can differ with time and usage [25,26], the probability of a failure due to stress is a function of the current deterioration level $X_{k}$ and the time $t_{k}$.

Let $Z_{k}$ be the system state indicator. If $Z_{k}=0$, the system is in a good state and $Z_{k}=1$ indicates a failed system. The failure due to shock for a non maintained system is given by the following conditional probability of a shock given the value of $X_{k}$, the magnitude $Y_{k}, t_{k} \geq 1$ :

$$
\left\{\begin{array}{l}
P\left(Z_{k}=1 \mid X_{k}=x<L, Y_{k}<\lambda\right)=1-e^{-a x-b t_{k}} \\
P\left(Z_{k}=1 \mid X_{k}=x<L, Y_{k} \geq \lambda\right)=1-F_{A} e^{-a x-b t_{k}}
\end{array}\right.
$$

where $a$ is the scale parameter, $b$ represents the sensitivity of the system to vibrations. 
As mentioned previously, the system condition can have some effects on the environmental stress, especially on the internal operating conditions. Let assume that a system failure due to an excessive deterioration or a shock increases continuously the stress intensity in average. In case of failure, a drift coefficient $\mu$ is introduced in the intensity stress process. Hence, the new process $\left(Y_{k}\right)_{k \in \mathbb{N}}$ after a failure is a Brownian motion with drift [27].

$\left(Y_{k}\right)_{k \in \mathbb{N}}$ represents in the example of the rollers the vibration amplitude. In fact, an increase in vibration can be associated with deteriorated states and thus a detection of a drift in vibration could prevent failures.

The two failure mechanisms are sketched in Figure 1. The effect of a failure in the two cases on the stress intensity process is also illustrated in the representation of $\left(Y_{k}\right)$. Finally, a system with a competing failure process is considered, the conditional probability of failure given the value of $X_{k}$ and/or $Y_{k}$ for $t_{k} \geq 1$ is obtained by adding to the set of equations (1) the probability of failure due to an excessive deterioration level:

$$
\left\{\begin{array}{l}
P\left(Z_{k}=1 \mid X_{k}=x \geq L\right)=1 \\
P\left(Z_{k}=1 \mid X_{k}=x<L, Y_{k}<\lambda\right)=1-e^{-a x-b t_{k}} \\
P\left(Z_{k}=1 \mid X_{k}=x<L, Y_{k}>\lambda\right)=1-F_{A} e^{-a x-b t_{k}}
\end{array}\right.
$$

\subsection{Reliability function}

Let $f_{\lambda}^{(k)}$ be the probability density function of the deterioration increment after $k$ periods of time of a non-failed system subject to deterioration and stress. $f_{\lambda}^{(k)}$ is given by:

$$
f_{\lambda}^{(k)}(x)=\left((1-p) F_{A}+p\right)^{k} \frac{\alpha^{k}}{a^{k-1}(k-1) !}\left(1-e^{-a x}\right)^{k-1} e^{-\alpha x} \prod_{j=1}^{k} e^{-b j}
$$

with $p=P\left(Y_{k}<\lambda\right)=\Phi\left(\frac{\lambda-m}{\sigma}\right) ; \Phi(\cdot)$ is the cumulative distribution function of the normal distribution.

The reliability of the unmaintained system at time $t_{k}=k \Delta t$ can be obtained directly by integrating $f_{\lambda}^{(k)}$ between 0 and $L$. The reliability $R\left(t_{k}\right)$ is given by:

$$
\begin{aligned}
R\left(t_{k}\right) & =\int_{0}^{L} f_{\lambda}^{(k)}(x) e^{-a x} d x \\
& =\left((1-p) F_{A}+p\right)^{k} \int_{0}^{L} \frac{\alpha^{k}}{a^{k-1}(k-1) !} e^{-(\alpha+a) x}\left(1-e^{-a x}\right)^{k-1} \prod_{j=1}^{k} e^{-b j} d x
\end{aligned}
$$


The curves in Figure 2 represent the reliability function (cf. Equation (4)), the associated probability of failure due to the different failure mechanisms (shock and excessive deterioration level) and the reliability without any stress so with a probability of failure due to shocks equal to 0 . This figure illustrates the impact of the "stress-failure" process on the reliability of the system.

\section{Definition and evaluation of the maintenance policy}

This section presents the maintenance decision framework which corresponds to a "control limit policy" $[28,29]$. First, the structure of the maintenance policy is presented to define when an inspection or a replacement should be implemented. Then, the mathematical expressions of the associated long-run maintenance cost per unit of time are developed to optimize the maintenance decision.

\subsection{Structure of the maintenance policy}

The maintenance policy is based on the failure model described in the previous section. Moreover, failures are assumed to be non-obvious, an inspection is required to know the system state. Two types of inspection are available, an inspection of the deterioration level (X-inspection) and an inspection of the system state (Z-inspection). The maintenance policy also offers the possibility of replacements either preventive or corrective. Finally, a routine maintenance action can be performed to reduce the impact of the stressful environment.

\subsubsection{X-inspection}

The cumulative deterioration level $X_{k}$ can be observed only through costly inspections. Let $c_{i x}$ be the unitary inspection cost. Even if non-periodic inspection strategies are optimal [24] from a mathematical point of view, their implementation in an industrial context remains difficult and a periodic strategy is more frequently used, thus a periodic age-based (versus time-based) strategy is proposed. The first inspection after a potential replacement of failed system is scheduled $\tau$ time units after the restarting time and then the system is inspected at regular time intervals $(\tau, 2 \tau, \ldots)$ until the next replacement. The benefit of such an assumption is a reduced number of the decision parameters, only the inspection period $\tau$. This inspection is assumed to be perfect in the sense that it reveals the exact deterioration level $X_{k}$. 


\subsubsection{Z-inspection}

The stress variable $Y_{k}$ is continuously monitored, i.e. at each time $t_{k}$. A classical control chart for individual observations [30] is implemented to detect a potential failure by the observation of a drift in $Y_{k}$. If the value $Y_{k}$ is greater than the Upper Control Limit, $U C L$, a minimal inspection is performed at a given unitary cost $c_{i z}$. This minimal inspection reveals only if the system is failed or not, i.e. $Z_{k}=0$ or $Z_{k}=1$, but delivers no information on $X_{k}$. A minimal inspection is less expensive than an inspection on $X_{k}, c_{i z}<c_{i x}$. In this paper, the value of $U C L$ is not evaluated in order to minimize the number of false alarms $\left(Y_{k}>U C L\right.$ but the system is still functioning) and non-detections (the system is in the failed state but $Y_{k}<U C L$ ), but the value of $U C L$ should be optimized to balance the cost incurred by the unavailability time due to a failure (incurred by a non-detection) and the cost incurred by false alarms (the cost of a false alarm corresponds to the cost of the system state inspection, $c_{i z}$ ). The computations of the number of false alarms and non-detections are developed in Appendix A.1. It should be noted that if the system is in the failed state during this inspection and thus a replacement is implemented, the next X-inspection is planned $\tau$ time units after.

\subsubsection{Preventive and corrective replacement}

A replacement can take place to renew the system when it is failed (corrective replacement) or to prevent the failure (preventive replacement). A corrective replacement is performed when the system is observed in the failed state during an X-inspection or during a minimal Z-inspection. The unitary cost of a corrective replacement $c_{c}$ includes all the direct and indirect costs incurred by this maintenance action. Only the unavailability cost $c_{u}$ per unit of time the system is failed has to be added to $c_{c}$. The decision rule for a preventive replacement is a classical control limit rule: if $\xi$ is the preventive replacement threshold, a preventive replacement is performed during the inspection on $X_{k}$ if the deterioration level belongs to the interval $(\xi, L)$. Let $c_{p}$ be the preventive replacement cost $\left(c_{p}<c_{c}\right)$. Replacements are assumed to be instantaneous.

\subsubsection{Routine maintenance action}

A routine maintenance action has to be implemented to reduce the impact of the stress on the system modeled by a decreasing in the failure hazard function. This maintenance action would not correspond to a minimal repair.

Routine maintenance is assumed to be instantaneous. In the example of the tramway, this operation can be e.g. the lubrication of the rails. The effec- 
tiveness of this action decreases with time and a new operation has to be scheduled. A periodic scheme is proposed for this operation, $\delta$ is the time between two successive actions. Let $c_{m}$ be the unitary operation cost (in the example, this cost would also include the tramway immobilization due to the unavailability of the track). Let $r_{k}$ be the time passed since the last action. We have $r_{k}=t_{k}-\delta$. $\left[\frac{t_{k}}{\delta}\right]$ where $[\cdot]$ is the integer part function. To model the effect of this maintenance on the system, $r_{k}$ is introduced in the conditional probabilities equations (1) instead of $t_{k}$ and thus the following system of equations is obtained:

$$
\left\{\begin{array}{l}
P\left(Z_{k}=1 \mid X_{k}=x<L, Y_{k}<\lambda\right)=1-e^{-a x-b r_{k}} \\
P\left(Z_{k}=1 \mid X_{k}=x<L, Y_{k}>\lambda\right)=1-F_{A} e^{-a x-b r_{k}}
\end{array}\right.
$$

Figure 3 represents the influence of the routine maintenance period, $\delta$, on the reliability and on the different probabilities of failure. The Figure 3(a) highlights the impact of the routine maintenance period on the reliability. The different curves are superimposed until $t_{1}=1$ the time of the routine maintenance in the case $\delta=1$. At $t_{2}=2$ the impact of the routine maintenance $(\delta=1)$ on the reliability is visible and leads to an increase in the reliability of $4.9 \%$ when compared to the cases $\delta=100$ and $\delta=3$. The curves for $\delta=3$ and $\delta=100$ are superimposed until $t_{3}=3$ the time of the first routine maintenance in the case $\delta=3$. At $t_{4}=4$ the reliability on the case of $\delta=3$ is improved of $17.4 \%$ when compared to $\delta=100$. The routine maintenance action slows down the decrease of the reliability each time it is performed, see the reliability curve on Figure $3(\mathrm{a})$, at times $t_{3}, t_{6}, \ldots$

The curves presented in Figure 3(b) illustrate the impact of the routine maintenance on the probability of failure due to shock. As previously mentioned, the three curves are superimposed until $t_{1}=1$ and then the curves which correspond to $\delta=3$ and $\delta=100$ are superimposed until $t_{3}=3$. On the curves which correspond to $\delta=3$, irregularities are visible at $t_{3}=3 ; t_{6}=6 ; t_{9}=9$ times of the routine maintenance.

The curves presented in Figure 3(c) illustrate the impact of the routine maintenance on the probability of failure due to an excessive deterioration level. The routine maintenance has an impact only on the probability of failure due to shock (cf. Equation (1)), the probability of failure due to an excessive deterioration level is not influenced by this maintenance action, the shape of the curves is always the same (all the curves are smooth) and only the proportion of failure due to this failure mechanism changes.

\subsubsection{Maintenance decision variables}

Finally, the maintenance decision parameters which should be optimized are: 
- $\tau$ : the inspection period which allows to balance the cumulative inspection cost and the early detection or the prevention of a failure;

- $\xi$ : the preventive maintenance threshold which allows to set the ratio between preventive and corrective replacements;

- UCL: the upper limit control which balances false alarms and failure detection;

- $\delta$ : the maintenance action period which allows for a reduction of the probability of shock.

An illustration of the evolution of a maintained system and of the maintenance decision is presented in Figure 4.

\subsection{Long-run average cost per time unit}

This section is presents the mathematical framework for evaluating the performance of the proposed maintenance policy with respect to the long-run maintenance cost per unit of time. The optimization criterion is a function of the maintenance decision parameter vector $\Xi=(\tau, \xi, U C L, \delta)$, the system deterioration characteristics $\theta=\left(a, b, F_{A}, \alpha, m, \mu, \sigma, \lambda, L\right)$ and the different maintenance costs.

The expected cumulative maintenance cost up to time $t_{k}$ incurred by the successive actions on the system knowing $\theta$ the system characteristics vector is given by the following equation:

$$
\begin{aligned}
\mathbb{E}\left(C\left(t_{k}\right)\right)= & c_{i x} \cdot \mathbb{E}\left(N_{i x}\left(t_{k}\right)\right)+c_{i z} \cdot \mathbb{E}\left(N_{i z}\left(t_{k}\right)\right)+c_{p} \cdot \mathbb{E}\left(N_{p}\left(t_{k}\right)\right)+\left(c_{c}-c_{p}\right) \cdot \mathbb{E}\left(N_{c}\left(t_{k}\right)\right) \\
& +c_{u} \cdot \mathbb{E}\left(D_{u}\left(t_{k}\right)\right)+c_{m} \cdot \mathbb{E}\left(N_{m}\left(t_{k}\right)\right)
\end{aligned}
$$

where:

- $\mathbb{E}($.$) represents the expected value knowing \theta$;

- $N_{i x}\left(t_{k}\right)$ (respectively $N_{i z}\left(t_{k}\right)$ ) is the number of X-inspections (respectively Z-inspections) performed before $t$;

- $N_{m}\left(t_{k}\right)$ is the number of routine maintenance performed before $t_{k}$;

- $N_{p}\left(t_{k}\right)$ (respectively $N_{c}\left(t_{k}\right)$ ) is the number of preventive (respectively corrective) replacement performed before $t_{k}$;

- $D_{u}\left(t_{k}\right)$ is the time that the system passed in failed state over $\left(0, t_{k}\right)$ knowing the system is considered as failed as soon as the system deterioration level exceeds its critical level $L$.

By using classical renewal arguments [31], this maintenance criterion can be expressed on a renewal cycle $S$ defined between two consecutive replacements 
because the system is considered as good as new after a replacement. Then, the expression of the long-run average maintenance cost per unit of time is :

$$
\begin{aligned}
C_{\infty}(\Xi ; \theta)= & \lim _{t_{k} \rightarrow \infty} \frac{C\left(t_{k}\right)}{t_{k}}=\frac{\mathbb{E}(C(S))}{\mathbb{E}(S)} \\
= & \frac{c_{i x} \mathbb{E}\left(N_{i x}(S)\right)+c_{i z} \mathbb{E}\left(N_{i z}(S)\right)+c_{p}+c_{m} \mathbb{E}\left(N_{m}(S)\right)+c_{u} \mathbb{E}\left(D_{u}(S)\right)}{\mathbb{E}(S)} \\
& +\frac{\left(c_{c}-c_{p}\right) \mathbb{E}\left(N_{c}(S)\right)}{\mathbb{E}(S)}
\end{aligned}
$$

The different expectations in Equation (7) can be evaluated by a function of the reliability of the maintained system at steady-state, $R_{m}\left(t_{k}\right)$. The difference between $R\left(t_{k}\right)$ (the reliability of the unmaintained system) and $R_{m}\left(t_{k}\right)$ results in the possibility of preventive replacement after inspection and thus reduces the possible states from which failure can occur. In order to determine $R_{m}\left(t_{k}\right)$ for all $t_{k} \in(0, S)$, two cases are identified:

- Case 1: no inspection on the system has been made before $t_{k}\left(t_{k}<\tau\right)$. The probability density function for the system to be in a good state is only a function of the observed deterioration level $x \in(0, L)$ at time $t_{k}$ and the consecutive shocks in $\left(0, t_{k}\right)$. Hence for $t_{k}<\tau$, we have:

$$
R_{m}\left(t_{k}\right)=\int_{0}^{L} f_{\lambda}^{(k)}(x) e^{-a x} d x
$$

- Case 2: at least one maintenance action has been made at $t_{k}\left(t_{k} \geq \tau\right)$. The probability density function for the system to have been never replaced is a function of the observed deterioration level $x \in(0, \xi)$ during the last inspection at time $\left(\left[t_{k} / \tau\right] \cdot \tau\right)$, (where [.] is the integer part function), the deterioration level reached since the last inspection $y \in(x, L)$ and the consecutive shocks in $\left(0, t_{k}\right)$. Hence for $t_{k} \geq \tau$, we have:

$$
R_{m}\left(t_{k}\right)=\int_{0}^{\xi} \int_{x}^{L} f_{\lambda}^{(k-[k / \tau] \cdot \tau)}(y-x) f_{\lambda}^{([k / \tau] \cdot \tau)}(x) e^{-a(y+x(k-[k / \tau] \cdot \tau))} d y d x
$$

The expected length of a regenerative cycle $\mathbb{E}(S)$ can be expressed by the four following exclusive scenarios:

- Scenario 1: A failure occurs between $(n \tau-\Delta t ; n \tau)_{n \in \mathbb{N}}$ (see Figure 5 ) and is detected during an $\mathrm{X}$-inspection at time $n \tau$, the system is replaced at this time.

- Scenario 2: A failure occurs between two inspections at time $t_{i} \in((n-$ 1) $\tau ; n \tau-\Delta t)_{n \in \mathbb{N}}$ (see Figure 5 ) and is not detected by the control chart but only during the following $\mathrm{X}$-inspection at $n \tau$.

- Scenario 3: A failure occurs between two inspections at time $t_{i} \in((n-$ 1) $\tau ; n \tau-\Delta t)_{n \in \mathbb{N}}$ (see Figure 5) and is detected by the control chart (a 
Z-inspection) before the next X-inspection.

- Scenario 4: A cycle ends by a preventive replacement during an X-inspection.

Hence we have:

$$
\begin{aligned}
& \mathbb{E}(S)=\sum_{i=0}^{\infty}[\underbrace{\mathbb{1}_{\{i>0\}} i \tau\left(R_{m}(i \tau-1)-R_{m}(i \tau)\right)}_{\text {Scenario } 1} \\
& +\underbrace{\sum_{j=i \tau+1}^{(i+1) \tau-1} i \tau\left(R_{m}(j-1)-R_{m}(j)\right) \prod_{k=j}^{(i+1) \tau-1} \mathbb{P}\left(Y_{k}<U C L \mid \text { fail. }\right)}_{\text {Scenario } 2} \\
& +\underbrace{\left.\sum_{j=i \tau+1}^{(i+1) \tau-1} j \mathbb{P}\left(Y_{j}>U C L \mid \text { fail. }\right)\left(R_{m}(j-1)-R_{m}(j)\right)\right]}_{\text {Scenario } 3} \\
& +\underbrace{\mathbb{1}_{\{j>i \tau+1\}} \sum_{k=i \tau+1}^{j-1} j\left(R_{m}(k-1)-R_{m}(k)\right) \prod_{w=k}^{j-1} \mathbb{P}\left(Y_{w}<U C L \mid \text { fail } .\right)}_{\text {Scenario } 3} \\
& +\underbrace{\left.\mathbb{1}_{\{i>0\}} i \tau\left(\int_{0}^{\xi} f_{\lambda}^{((i-1) \tau)}(x) d x-\int_{0}^{\xi} f_{\lambda}^{(i \tau)}(x) d x\right)\right]}_{\text {Scenario } 4}
\end{aligned}
$$

The computations of the other quantities are obtained similarly and are presented in appendix.

\section{Performance evaluation of the maintenance policy}

This section is devoted to illustrate the benefits of the proposed maintenance policy and compare its performance to classical policies. Numerical experiments are first proposed to highlight the convexity of the cost function and to analyze the influence of each maintenance decision parameter on the criterion. The economical performance of the proposed policy is illustrated by a comparison to classical approaches. Then, numerical studies are developed to illustrate the robustness of the policy when there are uncertainties on the system characteristics. All the numerical results are provided here by the use of R (www.r-project.org) software, specific programs are developed to numerically evaluate each expectation in equation (7) and the classical numerical gradient procedure provided by the $\mathrm{R}$ software is used. 


\subsection{Influence of the maintenance parameters}

To highlight the impact of the four decision parameters $(\tau, \xi, U C L, \delta)$ and the benefit of the combination of SPC and CBM, the performance of the maintenance policy is discussed when the decision parameters values change for a given set of maintenance data. This set of maintenance data is here a set of generic values which are arbitrarily fixed: $\alpha=5, a=0.05, b=0.025, F_{A}=$ $0.96, L=2, m=10, \mu=0.22, \sigma=1, \lambda=10.5, c_{c}=100, c_{p}=30, c_{i x}=2, c_{i z}=$ $0.5, c_{u}=25, c_{m}=4$. The cost curves presented in Figure 6 are obtained when two of the four maintenance decision parameters change and the two other parameters are fixed to their optimal value.

\subsubsection{Influence of $\tau$}

The influence of $\tau$ is illustrated in Figures 6(b), 6(d) and 6(e). An inspection allows for prevention and detection of a failure. When the inspection interval is too long, the main decision rule is restricted to the corrective replacement detected by the control chart. This information is illustrated in the flatness of the curve presented in Figure 6(e), the valley-shape of the curve in Figure 6(d) and in the increase of the cost value when $U C L$ increases in Figure 6(b). In the last case, the detection of a failure is thus delayed because of the lack of information on the system. When the inspection interval is small, a lot of information is available on the system with brief unavailability periods due to a failure. A monitoring policy is not relevant in this case (see the flatness of the curve when $\tau$ is small in Figure 6(b)).

\subsubsection{Influence of $\xi$}

The influence of $\xi$ is illustrated in Figures 6(a), 6(e) and 6(f). The preventive replacement threshold allows to prevent failure in case of sufficient information on the system. If this information is limited, the value of $\xi$ has no more influence on the criterion (see Figure 6(e) e.g. when $\tau>6$ ). When the frequency of the inspection is very high, the shape of the curve in Figure 6(e) illustrates the excessive cost of a too early preventive replacement. Moreover, the robustness of the optimal threshold value respectively with the control chart policy and the routine maintenance action period is illustrated by the valley-shape curves in Figure 6(a) respectively 6(f).

\subsubsection{Influence of $U C L$}

The influence of $U C L$ is illustrated in Figures 6(a), 6(b) and 6(c). As mentioned previously in section 4.1 .1 , the control chart allows to detect the failures. 
This conclusion is clearly illustrated in Figure 6(b) for high values in $\tau$ and $U C L$. Another aspect of the control chart illustrated in the decrease in the first part $(U C L<10)$ of the curve in Figure 6(b) when $\tau$ is very high $(\tau=10)$ is the balance with the detection of a failure and false alarms. The two other curves do not provide any relevant information on the impact of $U C L$ because, finally, a high level of information on the system state is available due to a relative low optimal value of the inspection interval $\tau^{*}=25$.

\subsubsection{Influence of $\delta$}

The influence of $\delta$ is illustrated in Figures 6(c), 6(d) and 6(f). The routine maintenance action allows to reduce the probability of failure due to a shock, thus it influences directly the reliability of the system. This information is illustrated in Figures 6(c), 6(d) and 6(f), whatever the value of the other parameters, the optimized value of $\delta$ is always the same. So, later in this paper, the value of this parameter will be fixed to this optimal value, $\delta^{*}=2$.

\subsection{Influence of the unit maintenance costs}

The global maintenance cost gathers inspection, preventive replacement, corrective replacement, routine maintenance and unavailability costs, see Equation (7). In this section, the consequences of the variations of those costs on the optimal maintenance policy parameters are investigated. Optimal maintenance parameters have been computed for several unit costs configurations. An example of the evolution of the optimal preventive threshold, the optimal upper control limit and the optimal inspection period as a function of the cost of the inspection on $X$ is given in Figure 7. A low cost of the inspection on $X$ causes a low inspection period $\left(\tau=1\right.$ for $\left.c_{i x}=1\right)$. Hence as long as no replacement is triggered, inspections are scheduled as frequently as possible. The proposed policy is closed to a systematic inspection/replacement one (cf. Section 3.4.2).

The value of the inspection period $\tau$ increases with $c_{i x}$, but in order to balance the lost due to the increasing value of $\tau, \xi$ and $U C L$ decrease. When $\tau$ becomes really high, the upper limit control allows for detection of a failure between two X-inspections and thus, this proposed policy is closed to a corrective replacement one: only a corrective replacement can be implemented as soon as a failure is detected by the monitoring of the stress covariate $Y_{k}$ (cf. Section 3.4.2).

Other numerical experiments have revealed the influence of the other maintenance costs. The inspection period $\tau$ and the preventive threshold $\xi$ increase with $c_{p}$, whereas $U C L$ decreases. A corrective replacement has to be preferred to a costly preventive replacement provided that the unavailability time is 
weakest as possible thanks to the value of the upper limit control. The upper control limit increases with $c_{c}$ and $c_{i z}$ so, in both of this cases the proposed policy tends to an inspection/replacement policy. The three maintenance parameters decrease with $c_{u}$ and the policy tends again to an inspection/replacement policy.

In a general way, it is noteworthy that an empirical choice of the maintenance parameters as an exclusive function of the unit costs is quite difficult to obtain. The separation of their influence remains possible only with some particular configurations. The last point emphasizes the need for an optimization procedure.

\subsection{Comparison with classical policies}

The economical performance of the proposed policy (hereafter denoted Policy 0 ) is illustrated by a comparison to classical approaches. The following three classical policies are considered.

- Policy 1 - periodic inspection/replacement. The deterioration level of the system is periodically inspected with a periodicity $\tau$ and the system is preventively replaced if its deterioration is found to be greater than $\xi$. In case of failure, the system is correctively replaced as soon as the failure is detected by the inspection on $X$.

- Policy 2 - control chart. Only a corrective replacement (cost $c_{c}$ ) could be implemented as soon as a failure is detected when the stress covariate $Y_{k}$ becomes greater than $U C L$.

- Policy 3 -block replacement policy. No inspection is performed. The system is replaced every time $T$. The replacement time has to be optimized to balance the system unavailability and the maintained system lifetime.

\subsubsection{Performance of the proposed maintenance policy when system charac- teristics vary}

The economic performance of each policy is evaluated when the system characteristics vary. All the results presented in this study are obtained for the optimized values of the decision parameters, only the maintenance action pe$\operatorname{riod} \delta$ is fixed (cf. results Section 3.1). Let denote $\left(\tau_{0}, \xi_{0}, U C L_{0}\right)$ (respectively $\left(\tau_{1}, \xi_{1}\right), U C L_{2}$ and $\left.T_{3}\right)$ the optimized decision parameters for Policy 0 (respectively Policy 1, 2 and 3).

The curves in Figure 8(a) are the respective representation of the optimized cost criterion calculated for the different policies. They are obtained when the scale parameter varies for 0 to 0.09 . All the other system characteristics are fixed. From the analysis of the curves, we can conclude that: 
- Policy 0 is always the policy which minimizes the cost criterion.

- When $a$ tends to 0, Policy 0 tends to Policy 1 . The gap between Policy 0 and 1 when $a=0$ is due to $U C L$ which permits to detect failure between the inspections on $X$.

- When $a$ increases Policy 0 tends to Policy 2. If the scale parameter would continue to increase, Policy 0 would tend to Policy 2. The probability of failure due to a shock becomes so big that only corrective replacements should be performed when a failure is detected by the drift in $Y_{k}$.

The curves in Figure 8(b) represent respectively the optimized cost criterion calculated for the different policies when the deterioration speed varies from 1 to 10. For a low deterioration speed, the probability of failure is mainly due to an excessive deterioration level, and the conditional maintenance policy used for $X_{k}$ allows to prevent failure. So Policy 0 tends to Policy 1 for a low deterioration speed. When the deterioration speed increases, the probability of failure due to a shock increases to, and Policy 0 tends to Policy 2.

The results are not presented here, but when the sensitivity to stress parameter and acceleration factor vary, Policy 1 is still the policy which minimizes the cost criterion and tends to one of the other policy in some extreme configurations.

\subsubsection{Performance of the proposed maintenance policy when maintenance costs vary}

The economic performance of each policy is evaluated when the unit maintenance cost vary. The curves in Figure 9 are the respective representation of the optimized cost criterion calculated for the different policies. They are obtained when the cost of the inspection on $X$ varies for 0 to 12 . All the other costs are fixed. Policy 0 is again the policy which minimizes the cost criterion. When the inspection cost $c_{i x}$ tends to 0 the inspection period decreases and tends to 1 . The failure is only detected during an inspection on $X$, thus Policy 0 tends to Policy 1. When the inspection cost $c_{i x}$ increases, Policy 0 tends to Policy 2. In order to reduce the influence of the increase in the inspection cost, the optimal inspection period increases whereas, in the same time, the optimal preventive threshold decreases and the optimal upper limit control decreases to reduce the unavailability of a failed system. For a high $c_{i x}$, the inspection period should be so long that only corrective replacements should be performed when a failure is detected by the drift in $Y_{k}$.

The same analysis has been made when the other maintenance costs vary and the results are the same: the proposed policy is always the policy which minimizes the cost criterion or in particular configuration the proposed policy tends to a classical policy.

The observations made in this section show the adaptability of the proposed 
policy to very different system characteristics. The proposed maintenance policy is demonstrated to be more general than the three others which can be, however, emulated by the proposed structure and can be considered as "limit" cases. As a result, the proposed policy shows all its interest in non-extreme configurations, i.e. when the behavior of the system is less predictable and when none of the unitary costs is negligible: in these cases, it is necessary to combine the condition-based maintenance policy and the statistical process control.

\subsection{Sensitivity analysis}

Collecting data in practical situation can be quite difficult in respect with the lack of data and the measurement variability. Hence, the quality of the different estimations is directly influenced by the data collection. The objective of this section is to analyze the robustness of the proposed maintenance policy when the estimations of the system characteristics are biased.

\subsubsection{Sensitivity analysis when the failure threshold (L) varies}

In practical application, it is not a simple task to identify with precision the value of the failure threshold, some errors of measurement or estimation can be made.

Figure 10 represents the influence of the error made on the failure threshold. Figure 10(a) proposes to compare the average cost obtained with the optimized maintenance policy from the value $L=2$ while an uncertainty on this parameter has been made (the full line) and the average optimized cost that we would have obtained if any uncertainty had been made (the dotted lines). Figure 10(b) illustrates the relative loss, expressed as a percentage, to this uncertainty. The proposed maintenance policy seems to be sufficiently robust with the uncertainty on the parameter $L$ : less than $0.65 \%$ of relative loss is obtained in the worst case for an uncertainty of $-10 \%$ on the parameter $L$.

\subsubsection{Sensitivity analysis of other system characteristics with the "One-Factor- At-A-Time" method}

The same studies as previously for the failure threshold have been made for the other system characteristics: the deterioration rate $(\alpha)$, the failure acceleration factor due to stress $\left(F_{A}\right)$, the scale parameter $(a)$, the sensitivity to stress $(b)$, the stress threshold $(\lambda)$, the nominal level of the stress $(m)$, the standard deviation of the stress $(\sigma)$ and the drift of the stress $(\mu)$. In all the cases, when an uncertainty is made on one parameter, we obtained less than $0.45 \%$ of relative loss in the worst case. By using the "One-Factor-At-A-Time" method, 
Table 1

Impact of the error made on the system characteristics vector on the maintenance cost

\begin{tabular}{|c|c|c|c|}
\hline Error made on the system characteristics vector & $-10 \%$ & 0 & $+10 \%$ \\
\hline Cost with uncertainties & 9.814 & 12.253 & 15.050 \\
\hline Optimal cost & 9.729 & 12.253 & 14.885 \\
\hline Relative lost compared to the optimal cost & $0.866 \%$ & 0 & $1.096 \%$ \\
\hline
\end{tabular}

the robustness of the model is highlighted when only one factor at a time varies for the given system characteristics vector $\left(a=0.05, b=0.025, F_{A}=0.96, \alpha=\right.$ $5, m=10, \mu=0.22, \sigma=1, \lambda=10.5, L=2)$.

\subsubsection{Sensitivity analysis of the system characteristics vector}

Previously, the "One-Factor-At-A-Time" method has been used, when one parameter varies, others remain constant. But in a practical case, the parameters vary simultaneously and the effect of each input on the output is not necessarily linear. In order to move away from the hypothesis of the linearity and to evaluate the effect an uncertainty of the system characteristics vector, the worst cases are considered, i.e. each parameter is set to its value corresponding to a relative error of $-10 \%$ or $+10 \%$. The results presented in Table 1 are obtained by using a Monte Carlo approach. Less than $1.1 \%$ of relative loss is obtained in the worst case.

Finally, given the results, the model seems to be robust to uncertainties made on the estimation of the system characteristics vector.

\section{Conclusion}

The main interests of this work are the two following points. Firstly, the construction of a failure model for continuously deteriorating systems subject to environmental influence which combines a degradation model well controlled, known and a model by shock for which uncertainties on the failure mechanism can be integrated. Secondly, the construction and the evaluation of maintenance policies for this model is proposed. We provide a simple and relevant maintenance policy which takes advantage of all the information available for the system state description. The combination of SPC and CBM provide an accurate decision-making tool for a system with several failure mechanisms. The associated stochastic model of the maintenance cost has been developed in order to assess the policy performance. The development of the cost model has been supported by the evaluation of the reliability of the maintained system. Numerical experiments have demonstrated the good performance of the 
proposed maintenance structure, namely its adaptability to different system characteristics, its lower cost when compared to classical maintenance policies and its robustness when system characteristics are estimated with uncertainty. Even if the proposed structure for maintenance decision has shown interesting performance when compared with classical maintenance policies, a lot of research work remains to be done. It could be interesting to relax some assumptions in order to consider more complicated situations, such as dependencies between the deterioration process and the stress covariate. Moreover, in general the system is not subject to a single stress phenomenon, but to several stress sources, so, it could be interesting to integrate several stresses in our model. Furthermore actually the stress is modeled by a discrete process but it could be interesting to model it with a continuous-time process.

\section{A Appendix}

\section{A.1 Evaluation of the expected number of inspections}

- $N_{i z}(S)$ is the number of inspections of $Z_{t_{k}}$. The expected number of inspections of $Z_{t_{k}}$ is given by the expected number of false alarms and the expected number of true alarms.

$$
\begin{aligned}
\mathbb{E}\left(N_{i z}(S)\right)= & \sum_{i=0}^{\infty} \underbrace{\left[\sum _ { j = i \tau + 1 } ^ { ( i + 1 ) \tau - 1 } \left[\mathbb{P}\left(Y_{j}>U C L \mid \text { nofailure }\right) R_{m}(j)\right.\right.}_{\text {false alarms }} \\
& +\underbrace{\left.\mathbb{P}\left(Y_{j}>U C L \mid \text { fail. }\right)\left(R_{m}(j-1)-R_{m}(j)\right) \prod_{w=j}^{i \tau-1} \mathbb{P}\left(Y_{w}<U C L \mid \text { failure }\right)\right]}_{\text {true alarms }}]
\end{aligned}
$$

- $\mathbb{E}\left(N_{i x}(S)\right)$ is the expected number of inspections of $X_{t}$ before $S$, it is given by:

$$
\begin{aligned}
\mathbb{E}\left(N_{i x}(S)\right)= & \sum_{i=0}^{\infty}\left[\mathbb{1}_{\{i>0\}} R_{m}(i-1)\right. \\
& \left.+\sum_{j=(i-1) \tau+1}^{i \tau+1}\left(R_{m}(j-1)-R_{m}(j)\right) \times \prod_{k=j}^{i \tau-1} \mathbb{P}\left(Y_{k}<\text { UCL } \mid \text { failure }\right)\right]
\end{aligned}
$$




\section{A.2 Evaluation of the expected number of maintenance actions}

- $\mathbb{E}\left(N_{m}(S)\right)$ the expected number of routine maintenance

$$
\begin{aligned}
\mathbb{E}\left(N_{m}(S)\right)= & \sum_{i=1}^{\infty}\left[\mathbb { 1 } _ { \{ i = \delta [ \frac { i } { \delta } ] \} } \left(R_{m}(i-1)\right.\right. \\
& \left.\left.+\sum_{j=(i-1) \tau+1}^{i \tau+1}\left(R_{m}(j-1)-R_{m}(j)\right) \times \prod_{k=j}^{i \tau-1} \mathbb{P}\left(Y_{k}<U C L \mid \text { failure }\right)\right)\right]
\end{aligned}
$$

- $\mathbb{E}\left(N_{c}(S)\right)$ is the expected number of a corrective replacements, it is given by:

$$
\begin{aligned}
\mathbb{E}\left(N_{c}(S)\right)= & \sum_{i=0}^{\infty}\left[\mathbb{1}_{\{i>0\}}\left(R_{m}(i \tau-1)-R_{m}(i \tau)\right)\right. \\
& +\sum_{j=(i-1) \tau+1}^{(i \tau-1)}\left(R_{m}(j-1)-R_{m}(j)\right) \times \prod_{k=j}^{i \tau-1} \mathbb{P}\left(Y_{k}<U C L \mid \text { failure }\right) \\
& +\sum_{j=i \tau+1}^{(i+1) \tau-1} \mathbb{P}\left(Y_{j}>\text { UCL } \mid \text { failure }\right) *\left[\left(R_{m}(j-1)-R_{m}(j)\right)\right. \\
& \left.\left.+\mathbb{1}_{\{j>i \tau+1\}} \sum_{k=i \tau+1}^{j-1}\left(R_{m}(k-1)-R_{m}(k)\right) \times \prod_{w=k}^{j-1} \mathbb{P}\left(Y_{w}<\text { UCL failure }\right)\right]\right]
\end{aligned}
$$

\section{A.3 Evaluation of the expected cumulative unavailability duration}

- $\mathbb{E}\left(D_{u}(S)\right.$, the expected value of the cumulative unavailability duration before $S$ is given by:

$$
\mathbb{E}\left(D_{u}(S)\right)=\sum_{i=0}^{\infty}\left[\sum_{j=i \tau+1}^{(i+1) \tau-1}\left(R_{m}(j-1)-R_{m}(j)\right) \prod_{k=j}^{(i+1) \tau-1} \mathbb{P}\left(Y_{k}<U C L \mid \text { failure }\right)\right]
$$

\section{References}

[1] C. Valdez-Flores and R.M. Feldman. A Survey of Preventive Maintenance Models for Stochastically Deteriorating Single-Unit Systems. Naval Research Logistics, 36:419-446, 1989.

[2] H. Wang. A Survey of Maintenance Policies of Deteriorating Systems. European Journal of Operational Research, 139:469-489, 2002. 
[3] I. Gertsbakh. Reliability Theory With Applications to Preventive Maintenance. Springer, 2000.

[4] P.A. Scarf. On the application of mathematical models in maintenance. European Journal of Operational Research, 99(2):493-506, 1997.

[5] J.M. van Noortwijk. A survey of the application of gamma processes in maintenance. Reliability Engineering and System Safety, doi: 10.1016 / j.ress.2007.03.019, 2007.

[6] H. Hong. Inspection and maintenance planning of pipeline under external corrosion considering generation of new defects. Structural Safety, 21:203-222, 1999 .

[7] A. Jeang. Tool replacement policy for probabilistic tool life and random wear process. Quality and Reliability Engineering International, 15:205-212, 1999.

[8] J.M. van Noortwijk, M. Kok, and R. Cooke. Optimal maintenance decisions for the sea-bed protection of the eastern-scheldt barrier. European Journal of Operational Research, 82(2):25-56, 1997.

[9] E. Deloux, B. Castanier, and C. Bérenguer. Combining statistical process control and condition-based maintenance for gradually deteriorating systems subject to stress. In Aven \& Vinnem, editor, Risk, Reliability and Societal Safety, Proceedings of ESREL 2007- European Safety and Reliability Conference 2007, Stavanger, Norway, 25-27 June 2007, pages 265-272. Taylor \& Francis, 2007.

[10] W.Q. Meeker and L.A. Escobar. Statistical Methods for Reliability Data. Wiley, New-York, 1998.

[11] N.D. Singpurwalla. Reliability and risk A Bayesian Perspective. Wiley, 2006.

[12] V. Bagdonavicius and M. Nikulin. Estimation in Degradation Models with Explanatory Variables. Lifetime Data Analysis, 7:85-103, 2000.

[13] A. Lehmann. Joint modeling of degradation and failure time data. In Degradation, Damage, Fatigue and Accelerated Life Models In Reliability Testing, ALT2006, Angers, France, pages 26-32, 2006.

[14] N.D. Singpurwalla. Survival in Dynamic Environments. Statisticals Science, 10(1):86-103, 1995.

[15] A.I. Yashin and K.G. Manton. Effects of Unobserved and Partially Observed Covariate Processes on System Failure: A Review of Models and Estimation Strategies. Stasticals Science, 12(1):20-34, 1997.

[16] E.A. Elsayed and C.K. Chan. Estimation of thin-oxide reliability using proportional hazards models. IEEE Transactions on Reliability, 39(3):329-335, 1990.

[17] J.M. van Noortwijk, M.J. Kallen, and M.D. Pandey. Gamma processes for timedependent reliability of structures. In K. Kolowrocki, editor, Advances in Safety and Reliability, Proceedings of ESREL 2005 - European Safety and Reliability 
Conference 2005, Tri City (Gdynia-Sopot-Gdansk), Poland, 27-30 June 2005, 2005.

[18] W. Wang. A two-stage prognosis model in condition based maintenance. European Journal of Operational Research, 182(3):1177-1187, 2007.

[19] Y. Zhan and C.K. Mechefske. Robust detection of gearbox deterioration sing compromised autoregressive modeling and Kolmogorov-Smirnov test statitstic. Part II: Experiment and application. European Journal of Operational Research, 21:1983-2011, 2007.

[20] M. Ben-Daya and M.A. Rahim. Effect of maintenance on the economic design of x-control chart. European Journal of Operational Research, 120:131-143, 2000.

[21] C.R. Cassady, R.O. Bowden, L. Liew, and E.A. Pohl. Combining preventive maintenance and statistical process control: a preliminary investigation. IIE Transactions, 32:471-478, 2000.

[22] T. G. Yeung, C. R. Cassady, and K. Schneider. Simultaneous optimization of /x/-bar control chart and age-based preventive maintenance policies under an economic objective. Working paper, 2006.

[23] K. Linderman, K.E. McKone-Sweet, and J.C. Anderson. An integrated systems approach to process control and maintenance. European Journal of Operational Research, 164:324-340, 2005.

[24] B. Castanier, C. Bérenguer, and A. Grall. A sequential condition-based repair/replacement policy with non-periodic inspections for a system subject to continuous wear. Applied Stochastic Models in Business and Industry, 19(4):327$347,2003$.

[25] N.D. Singpurwalla and S. Wilson. Failure Models Indexed by Two Scales. Advances in Applied Probability, 30:1058-1072, 1998.

[26] S.C. Yang. A Bivariate Renewal Process and its Application in Maintenace Policies. $\quad \mathrm{PhD}$ thesis, Faculty of Virginia Polytechnic Institute and State University, 1999.

[27] S.M. Ross. Stochastic Processes. Wiley, second edition, 1996.

[28] S.K. Park. Optimal wear-limit replacement with wear-dependent failures. IEEE Transactions on Reliability, 27:293-294, 1988.

[29] D. Zuckerman. Optimal replacement policy for the case where the damage process is a one-side Lévy process. Stochastic Processes and their Applications, 7(141-151), 1978.

[30] I.W Burr. Statistical quality control methods. Marcel Dekker, 1976.

[31] S. Asmussen. Applied Probability and Queues, Wiley Series in Probability and Mathematical Statistics. Wiley, 1987. 


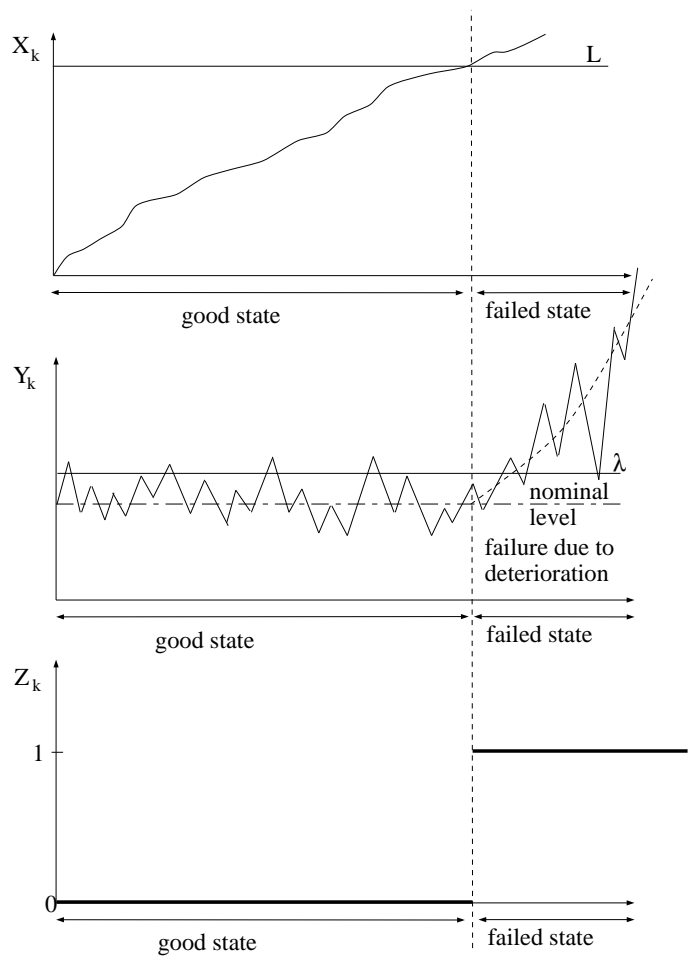

(a) Failure due to an excessive deterioration level, $X(t)>L$

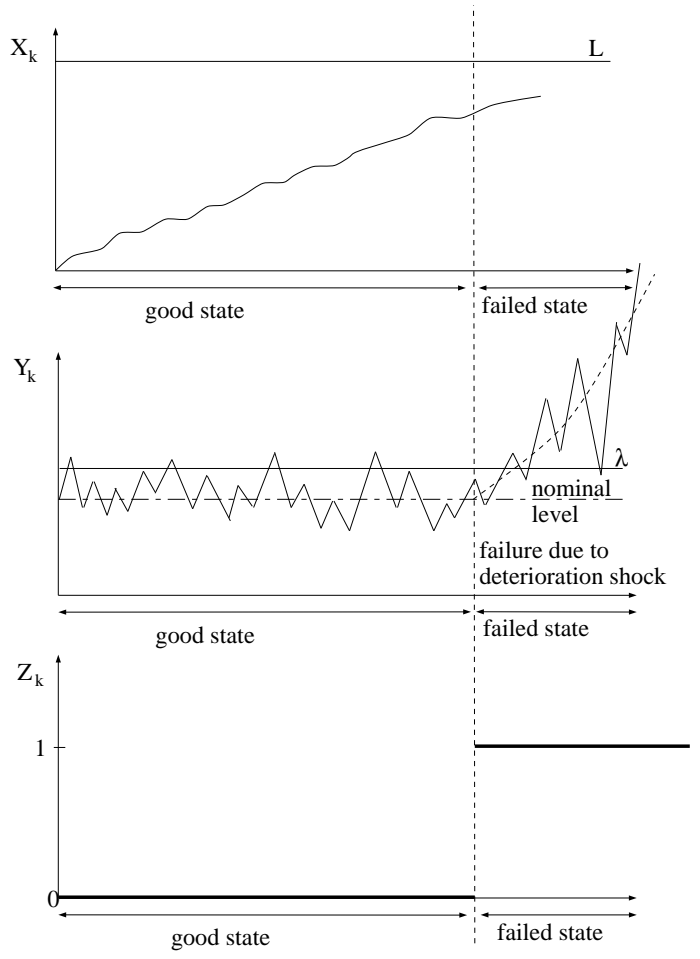

(b) failure due to shock

Fig. 1. Evolution of the deterioration process, stress process and system state of a non-maintained system 


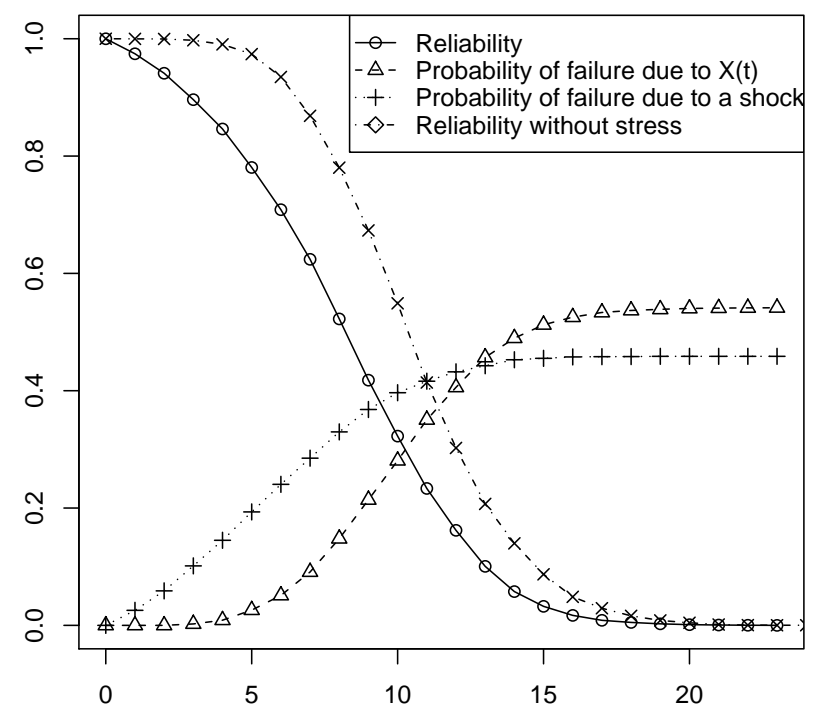

Fig. 2. Reliability and probability of failure due to an excessive deterioration or due to a shock with $\alpha=5 ; a=0.05 ; F_{A}=0.96 ; b=0.025 ; L=2$ 


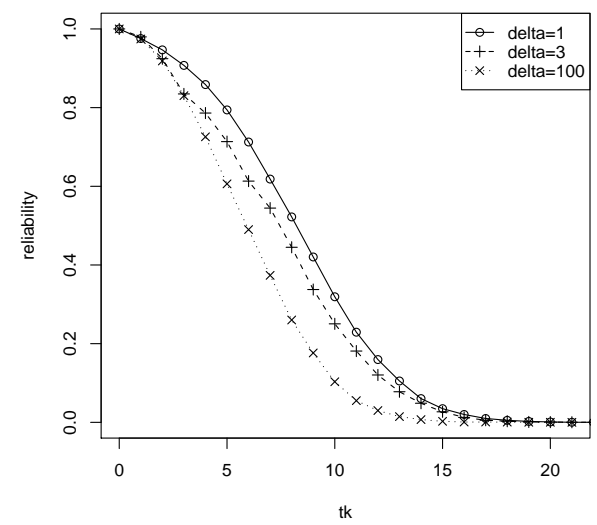

(a) Influence of $\delta$ on the reliability

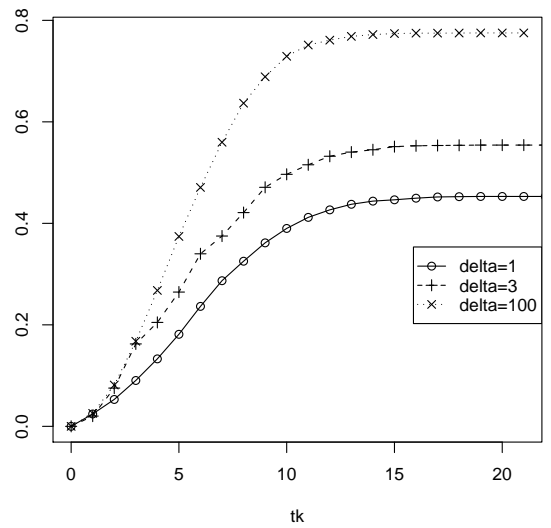

(b) Influence of $\delta$ on the conditional probability of failure due to shock at $t_{k}$ given $Z_{t_{k-1}}=0$

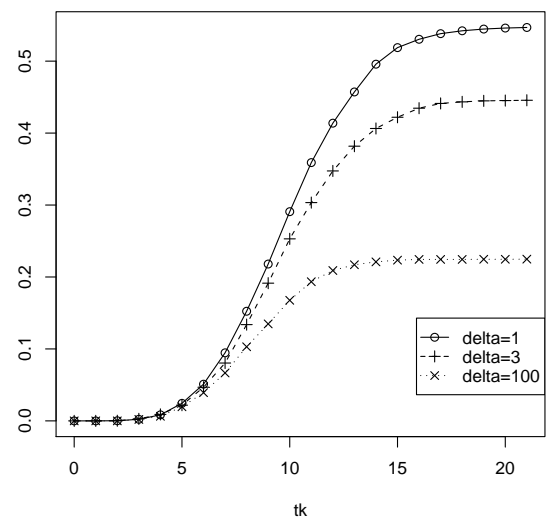

(c) Influence of $\delta$ on the conditional probability of failure due to $X_{t_{k}}$ at $t_{k}$ given $Z_{t_{k-1}}=0$

Fig. 3. Illustration of the influence of routine maintenance period $\delta$ on the reliability and the different probabilities of failure 


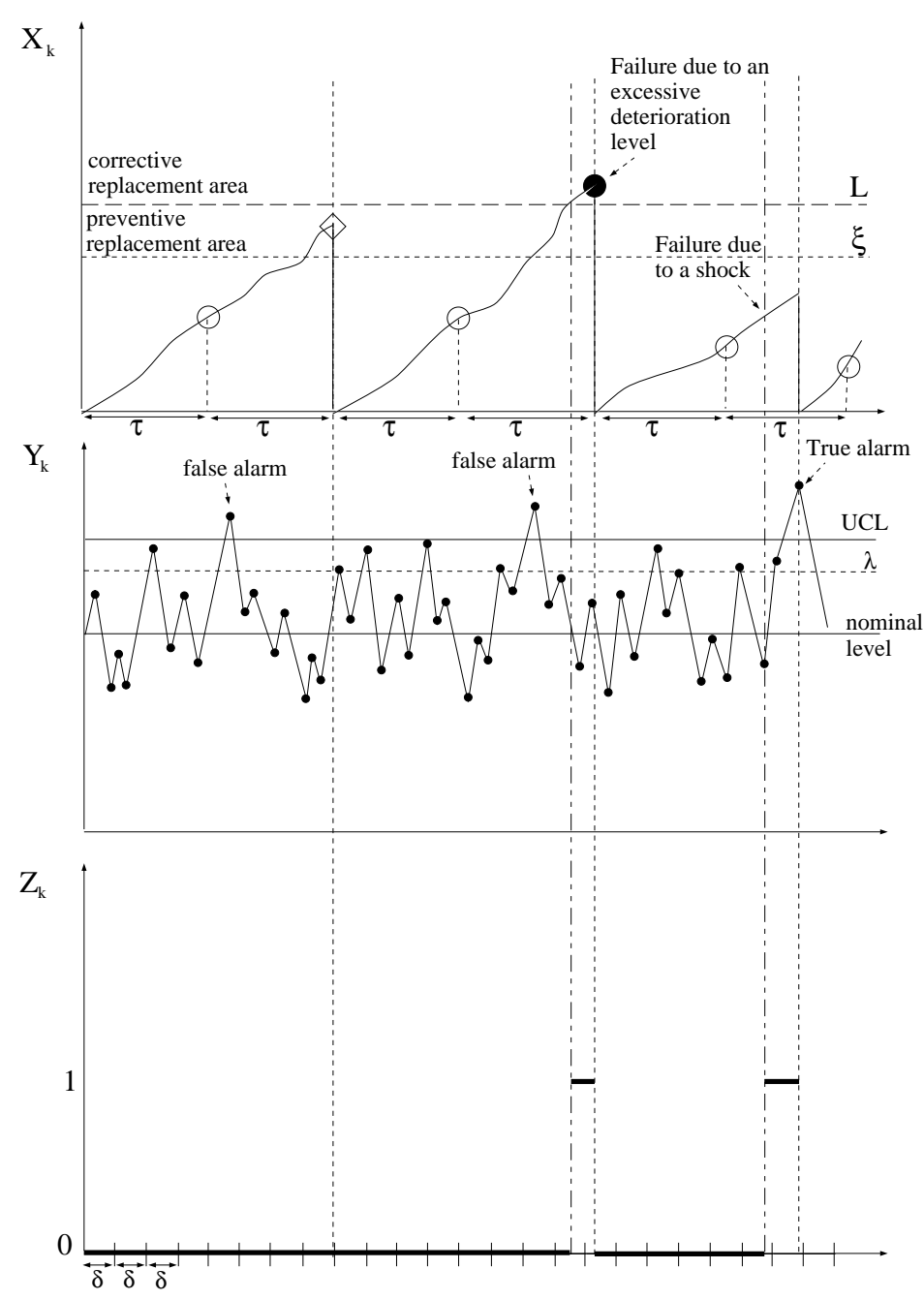

Fig. 4. Example of the evolution of the deterioration process, the stress process and the system state of a maintained system: $\bigcirc$ X-inspection; $\bullet Z$-inspection; $\diamond$ preventive replacement; corrective replacement

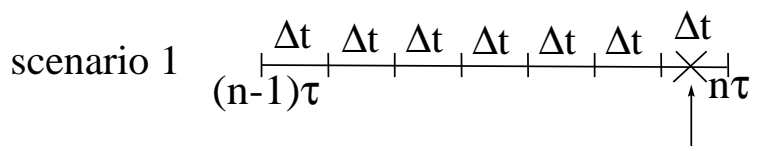

Failure

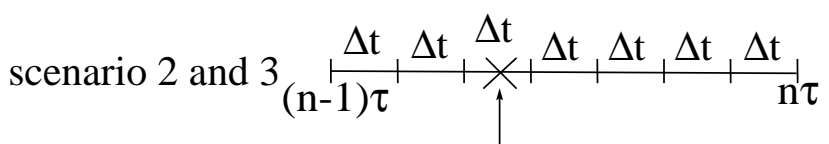

Failure

Fig. 5. Scale allowing to locate the three scenarios 

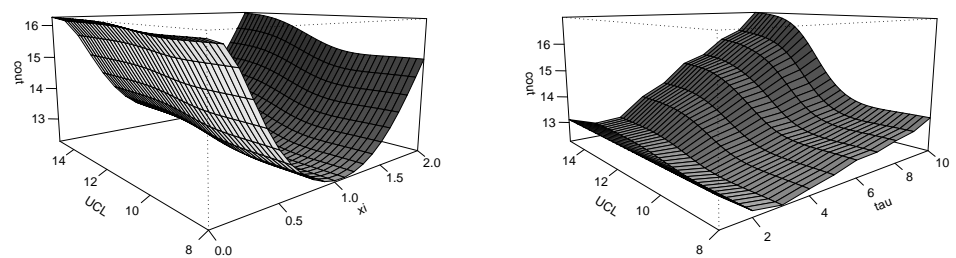

(a) Influence of $U C L$ and $\xi$ on (b) Influence of $U C L$ and $\tau$ on the cost $(\tau$ and $\delta$ fixed) the cost ( $\xi$ and $\delta$ fixed)
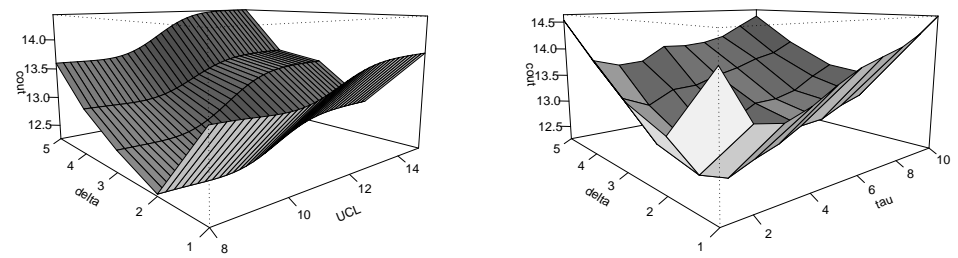

(c) Influence of $\delta$ and $U C L$ on (d) Influence of $\delta$ and $\tau$ on the the cost ( $\tau$ and $\xi$ fixed) cost ( $\xi$ and $U C L$ fixed)
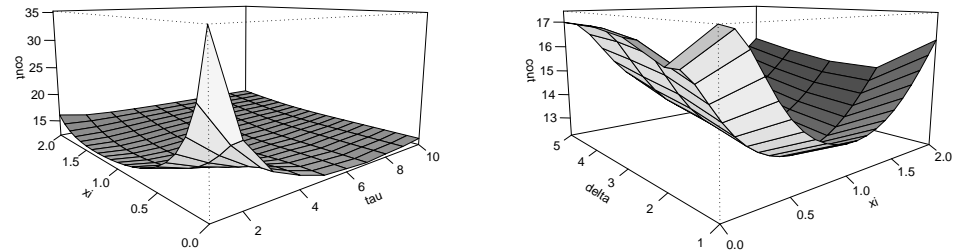

(e) Influence of $\xi$ and $\tau$ on the (f) Influence of $\delta$ and $\xi$ on the cost ( $\delta$ and $U C L$ fixed) cost ( $\tau$ and $U C L$ fixed)

Fig. 6. Cost evolution according to $\xi, \tau, U C L, \delta$ 

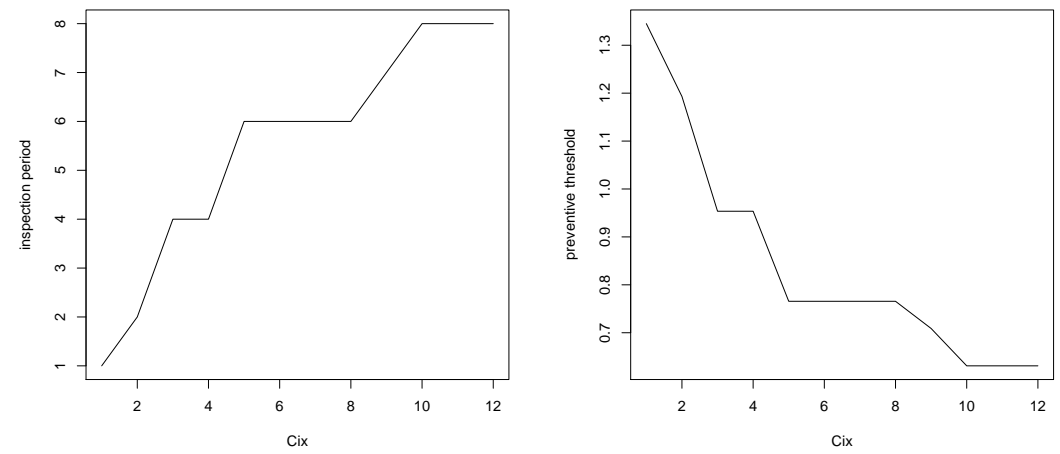

(a) influence on the inspection (b) influence of the preventive period threshold

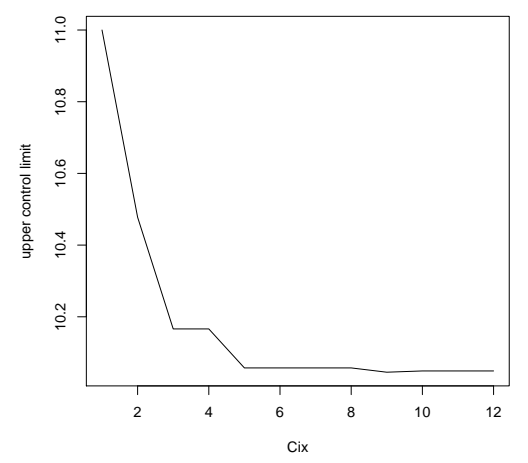

(c) influence of the upper limit control

Fig. 7. Influence of the cost of the inspection of $X_{t}$ 


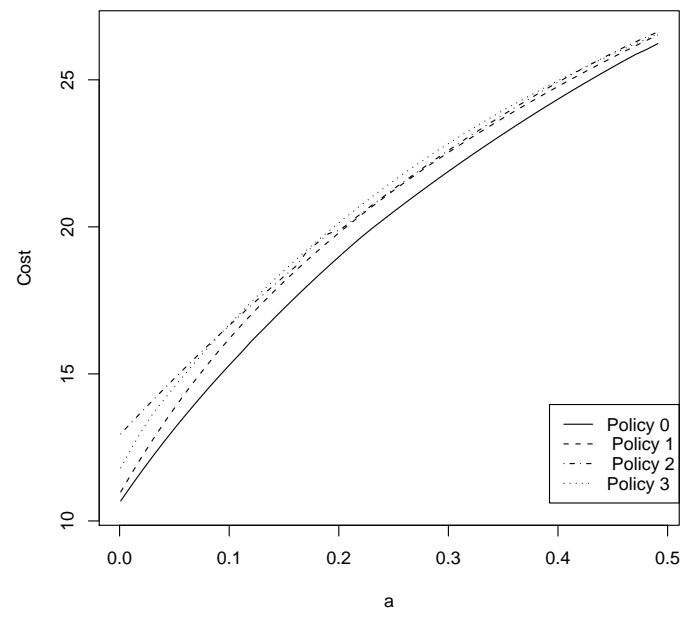

(a) Influence of the scale parameter

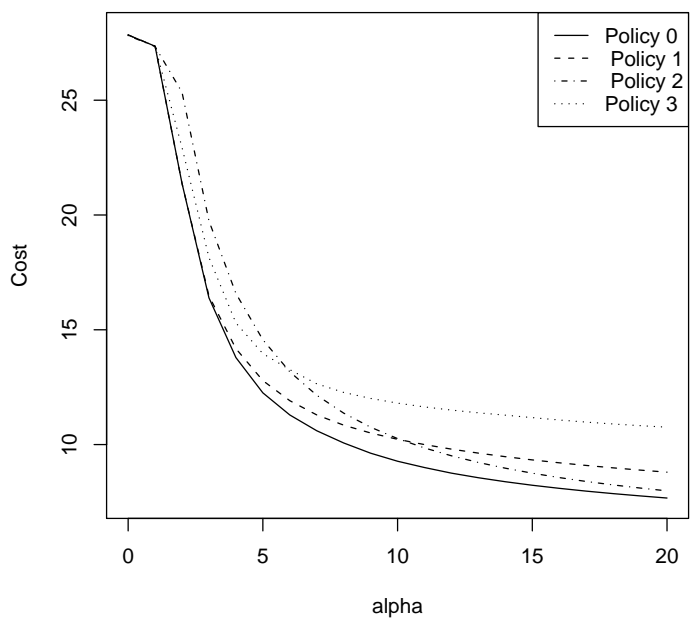

(b) Influence of the deterioration speed

Fig. 8. Influence of the system characteristics 


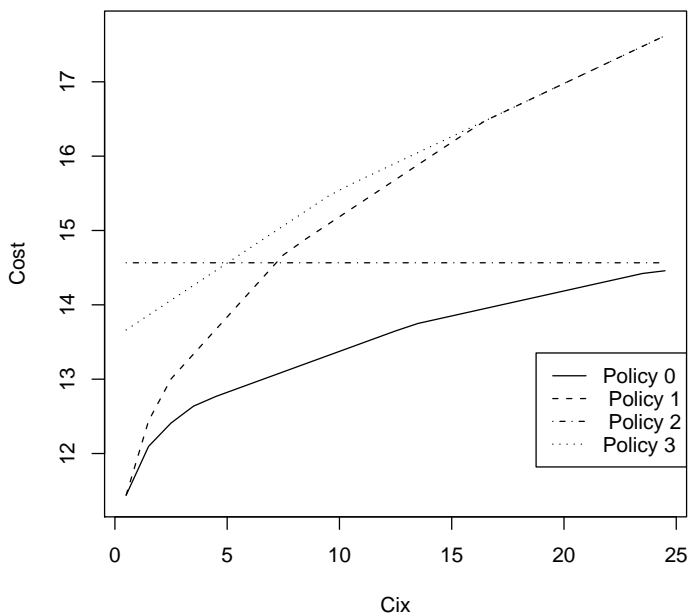

Fig. 9. Influence of the system characteristics: cost variation when $c_{i x}$ increases 


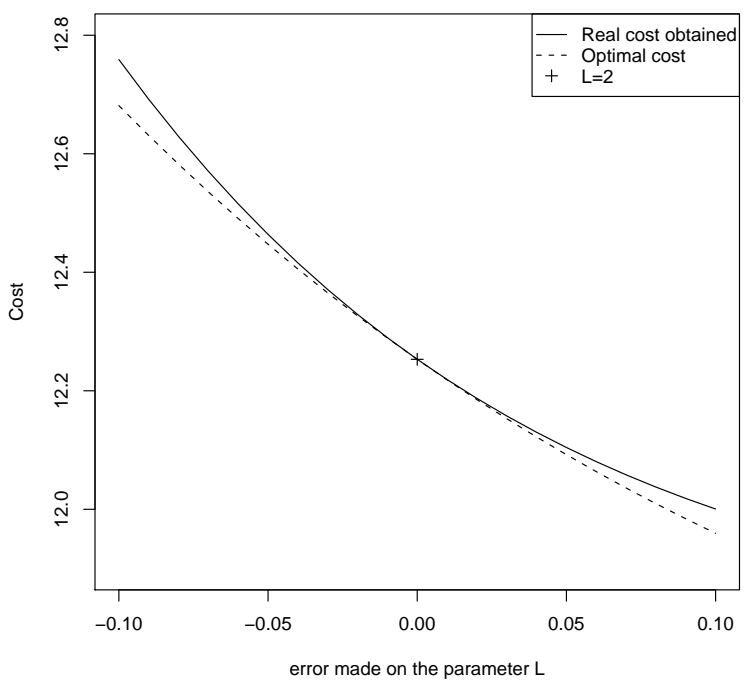

(a) Comparison of the average cost obtained and the optimized cost

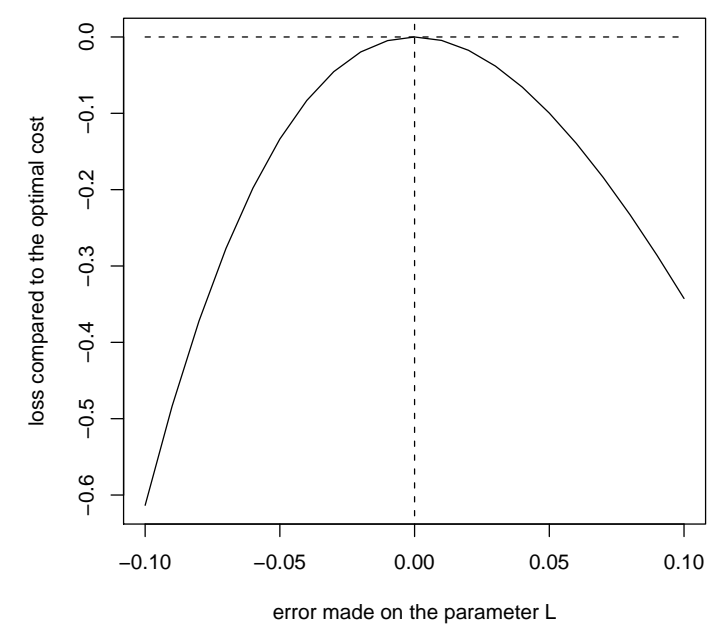

(b) Loss compared to the optimal cost

Fig. 10. Influence of the error made on the failure threshold: $c_{c}=100 ; c_{p}=30 ; c_{i x}=2 ; c_{i z}=0.5 ; c_{u}=25 ; c_{m}=4 ; \alpha=5 ; a=0.05 ; F_{A}=0.96 ; b=0.025$ 